\title{
Radius of convergence of a power series
}

\author{
TODOR D. TODOROV
}

We derive two simple and memorizable formulas for the radius of convergence of a power series which seem to be appropriate for teaching in an introductory calculus course. 


\section{Introduction}

Every proper power series (which is not a finite sum) with real coefficients can be uniquely presented in the form $\sum_{n=0}^{\infty} c_{n}\left(x-x_{0}\right)^{\nu_{n}}$, where $\left(c_{n}\right)$ is a sequence in $\mathbb{R}$ such that $c_{n} \neq 0$ for all $\mathrm{n}$, and $\left(\nu_{n}\right)$ is a strictly increasing sequence in $\mathbb{N}_{0}=\{0,1,2, \ldots\}$ such that $\lim _{n \rightarrow \infty}\left(\nu_{n}\right)=\infty$. Here $\mathbb{R}$ denotes, as usual, the set of real numbers. From the Ratio Test it follows that the radius of convergence $R$ of the series can be expressed by $R=\sup S$, where

$$
S=\left\{x \in \mathbb{R}: \lim _{n \rightarrow \infty}|| \frac{q+1}{c_{n}}|| \mid\left(x-\left.x_{0}\right|^{\nu_{n+1}-\nu_{n}}<1\right\}\right.
$$

In a typical calculus course we (ften tacitly) assume that the sequence of exponents $\left(\nu_{n}\right)$ is in the form $\nu_{n}=p n+q$ for some integers $p$ and $q$. Under this assumption the set in (1) converts into:

$$
S=\left\{x \in \mathbb{R}: \lim _{n \rightarrow \infty}\left|\frac{c_{n+1}}{c_{n}}\right|\left|x-x_{0}\right|^{p}<1\right\}
$$

Then we ask our students to solve the inequality (2) for $x$ and find the interior of the interval of convergence which is in the form $\left(x_{0}-R, x_{0}+R\right)$. The method works smoothly except (ironically) in the case when the series is convergent for all $x$. Indeed, in this case we have

$$
\lim _{n \rightarrow \infty}\left|\frac{c_{n+1}}{c_{n}}\right|=0
$$

and the set (2) converts to $S=\{x \in \mathbb{R}: 0 \leqslant 1\}$. Many students stumble at that point. We should not blame them for their failure; after all even an experienced mathematician might hesitate for a moment if forced to conclude that the set $\{x \in \mathbb{R}: 0<1\}$ is, actually, $\mathbb{R}$. Neither should we insist too much on the merits of our method based on the ratio test (advocating, for example, 'to keep the limit $\lim _{n \rightarrow \infty}$ in (2) passive'.

In this note we suggest an alternative: we derive two simple and memorizable formulas for the radius of convergence $R$ of power series. We suggest that the teaching of radius of convergence based on this formula has an advantage over the more conventional method based on ratio test and solving an inequality for the following two reasons: (a) our formula reduces the problem for finding the radius of convergence $R$ to calculating a limit in $\mathbb{R} \cup\{\infty\}$ only; (b) our method never requires solving artificial inequalities with 'missing variables' such as $0 \cdot\left|x-x_{0}\right|^{p}<1$.

\section{Ratio and root formulas}

Theorem 1. Let

$$
\sum_{n=m}^{\infty}\left(f_{n}\left(x-x_{0}\right)^{p n+q}\right.
$$

be a power series, where $x_{0}$ and $c_{n} \neq 0$ are real numbers, $m, p$ and $q$ are integers, such that $m \geq 0$ and $p n+q \geq 0$ for all $n$ (which implies $p>0$ ). Then:

(i) If 


$$
\lim _{n \rightarrow \infty}\left|\frac{c_{n}}{c_{n+1}}\right|(
$$

exists in $\mathbb{R} \cup\{\infty\}$, then the radius of convergence $R$ of the series is given by the formula:

(ii) If $\lim _{n \rightarrow \infty} \sqrt[n]{\left|c_{n}\right|}(\mathrm{ex}$

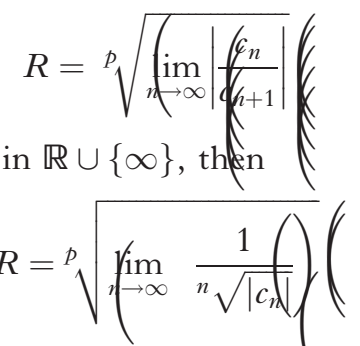

where we assume, by convention, that $\sqrt[1]{a} \notin a$ and $\sqrt[D]{\infty} \notin \infty$.

Proof. (i) By the ratio test, our series is absolutely convergent if and only if

$$
\lim _{n \rightarrow \infty}\left|\frac{a_{n+1}(x)}{a_{n}(x)}\right|<1
$$

where $a_{n}(x)=c_{n}(x-c)^{p n+q}$. On the other hand, we have:

$$
\begin{aligned}
& \lim _{n \rightarrow \infty}\left|\frac{f_{n+1}(x-c)^{p n+p+q}}{\mid c_{n}(x-c)^{p n+q}}\right|=\left.\lim _{n \rightarrow \infty}\left|\frac{c_{n+1}}{c_{n}}\right|\right|^{p}\left(|x-c|^{p}\right. \\
& \lim _{n \rightarrow \infty}\left|\frac{c_{n+1}}{c_{n}}\right|\left(|x-c|^{p}<1\right.
\end{aligned}
$$

which implies equation (3) (as required) since ${ }^{p} \sqrt{x}$ /s an increasing function of $\mathrm{x}$. The proof of (ii) is similar and we leave it to the reader.

Example 1. Consider the series

$$
\sum_{n=0}^{\infty} \frac{7^{n}(x+5)^{3 n+1}}{5 n+1}
$$

We have $p=3$ and

$$
c_{n}=\frac{7^{n}(x+5)^{3 n+1}}{5 n+1}
$$

Thus

$$
R=\sqrt[3]{\lim _{n \rightarrow \infty} \frac{5 n+6}{7(5 n+1)}}=\frac{2}{\sqrt[3]{7}}
$$

Example 2. Consider the series

$$
\sum_{n=1}^{\infty}\left(\frac{-1)^{n} x^{2 n-1}}{(2 n-1) !}\right.
$$

We have $p=2$ and $c_{n}=(-1)^{n} /(2 n-1)$ ! and our formula (3) gives 


$$
R=\sqrt{\lim _{n \rightarrow \infty} \frac{(2 n+1) !}{(2 n-1) !}}=\sqrt{\lim _{n \rightarrow \infty}[(2 n+1) 2 n]}=\sqrt{\infty} \neq \infty
$$

Remark. How restrictive are the conditions in Theorem 1? The best way to answer this question is to open your calculus textbook and check how many of the problems in the corresponding exercise section satisfy these conditions. You should not be surprised if all problems satisfy our conditions. In particular all problems in [1] (Section 12.8, pp. 778-779) can be solved by one of our two formulas.

\section{Acknowledgments}

The author thanks Gary Epstein and Ivan Dimitrov for the useful critical remarks on an earlier version of the manuscript.

\section{References}

[1] Stewart, J., 1999, Calculus, 4th edition (Brooks-Cole Publishing Co.). 